\title{
Ginsenoside Rg1 prevents early diabetic retinopathy via reducing retinal ganglion cell layer and inner nuclear layer cell apoptosis in $\mathrm{db} / \mathrm{db}$ mice
}

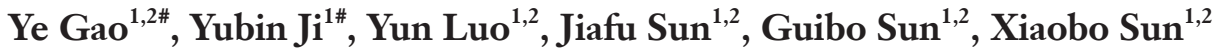 \\ ${ }^{1}$ College of Pharmacy, Harbin University of Commerce, Harbin 150076, China; ${ }^{2}$ Institute of Medicinal Plant Development, Peking Union Medical \\ College and Chinese Academy of Medical Sciences; Beijing Key Laboratory of Innovative Drug Discovery of Traditional Chinese Medicine (Natural \\ Medicine) and Translational Medicine; Key Laboratory of Bioactive Substances and Resource Utilization of Chinese Herbal Medicine, Ministry of \\ Education, Beijing 100193, China \\ Contributions: (I) Conception and design: Y Luo, X Sun; (II) Administrative support: G Sun, Y Gao; (III) Provision of study materials or patients: J \\ Sun, Y Gao; (IV) Collection and assembly of data: G Sun, Y Gao, J Sun; (V) Data analysis and interpretation: Y Gao, Y Luo; (VI) Manuscript writing: \\ All authors; (VII) Final approval of manuscript: All authors. \\ \#These authors contributed equally to this work. \\ Correspondence to: Yun Luo, Guibo Sun, Xiaobo Sun. Institute of Medicinal Plant Development, Chinese Academy of Medical Sciences \& Peking \\ Union Medical College, No. 151, Malianwa North Road, Haidian District, Beijing 100193, China. Email addresses: ly20040423@126.com; \\ sunguibo@126.com; sun_xiaobo163@163.com.
}

\begin{abstract}
Background: Diabetic retinopathy (DR), a diabetic vascular complication, is prone to developing into blindness. Ginsenoside Rg1 (GRg1), a major saponin in ginseng, exerts high anti-apoptotic activity.

Methods: This study aimed to explore the protective effects of GRg1 against diabetes-induced retinal damage. Measurements of blood glucose, blood lipids and vascular permeability were performed, as well as assessments of pathological changes, and the retinal thickness of each layer. Retinal cell apoptosis related protein expression levels were measured by immunofluorescence and western blot assays.

Results: Our data demonstrated that GRg1 effectively reduced blood glucose and triglyceride levels and maintained normal retinal permeability and physiological structure. GRg1 maintained the thickness of the ganglion cell layer (GCL) and the inner nuclear layer (INL) by reducing cell apoptosis.

Conclusions: These data strongly indicate that GRg1 prevents diabetic retinal changes by decreasing GCL and INL cell apoptosis. GRg1 may be a promising drug for early DR treatment.
\end{abstract}

Keywords: Diabetic retinopathy (DR); ganglion cell layer (GCL); inner nuclear layer (INL); apoptosis; ginsenoside $\operatorname{Rg} 1$ (GRg1)

Submitted Aug 23, 2019. Accepted for publication Dec 20, 2019.

doi: 10.21037/atm.2019.12.155

View this article at: http://dx.doi.org/10.21037/atm.2019.12.155

\section{Introduction}

With the rising frequency of obesity and improved detection of the disease, diabetic patients worldwide are predicted to grow to 629 million by 2045 (1). Numerous studies have demonstrated that diabetes mellitus induces diverse complications, such as diabetic retinopathy (DR), diabetic nephropathy and diabetic cardiovascular and cerebrovascular diseases, which have been the main causes of diabetes-associated morbidity and mortality (2). DR, a common diabetic microvascular complication, remains the leading cause of blindness in younger adults. DR begins with hyperglycemia and sustained oxidative stress or inflammation, which accelerate vascular leakage and retinal damage, and then induce macular edema and impaired vision (3). Clinically, DR can be alleviated by laser-induced photocoagulation, but this procedure may damage the 
retina (4). Moreover, injection of ranibizumab, an antivascular endothelial growth factor drug has been useful for some patients with macular edema or proliferative disease since it was approved by the FDA in 2017 (5). Unfortunately, the above DR treatments also bring adverse effects and economic burdens to the patients and their families (6). However, little is usable for the early stage of the disease. Facing the currently limited therapeutic options, we should find safe and effective drugs to treat early DR.

It has been widely accepted that high blood glucose and lipids are direct factors for the development of DR (3). These stimulations lead to retinal cell loss, which precedes microvascular changes that are characteristic of DR (7). Sustained vascular burden induces vascular permeability in diabetic retinas, which is another important characteristic of early DR (8). Therefore, retinal cell apoptosis and vascular permeability may be therapeutic targets for early DR treatment.

Recently, natural products have been a hotpot in anti-DR drug discovery $(9,10)$. The natural flavonoid galangin alleviates blood-retinal barrier damage by abrogating microgliatrigged oxidative stress injury via activating Nrf2 (11). Scutellarin alleviates blood-retina-barrier breakdown by abrogating retinal inflammatory responses and subsequent oxidative stress injury (12). Saponins, another type of compound, also have strong retinal protective effects. Notoginsenoside R1 attenuates high glucose-induced changes in the cellular redox state (13). Ginsenoside $\operatorname{Rg} 1$ (GRg1), a steroidal saponin, is abundant in ginseng, pseudoginseng and American ginseng. Recently, it was reported that GRg1 has strong anti-oxidative and hypoglycemic activities (14,15). Moreover, GRg1 ameliorates ER stressinduced apoptosis in streptozotocin-induced diabetic cardiomyopathy (16). No previous literature has studied the effect of GRg1 on diabetic retinal damage. The aim of this study was to explore the effects of GRg1 on DR in $\mathrm{db} / \mathrm{db}$ mice and to clarify whether the protective effects of GRg1 were mediated through anti-apoptotic mechanisms.

\section{Methods}

\section{Ethic statement}

All animal care procedures and interventions were consistent with the Guidelines and Policies for Animal Surgery provided by the Chinese Academy of Medical Sciences and Peking Union Medical College, Beijing, China
[SYXK(Jing) 2017-0020] and admitted by the Institutional Animal Use and Care Committee.

\section{Reagents}

GRg1 (purity 98\%) was obtained from the Shanghai Winherb Medical Technology Company (Shanghai, China). Dobesilate was purchased from Xi'an Lijun Pharmaceutical Co., Ltd. (Xi'an, China). Tropicamide phenylephrine eye drops were acquired from Shenyang Xingqi Pharmaceutical Co., Ltd. (Shengyang, China). Carbomer eye drops were obtained from Shandong Baushiren Furuida Pharmaceutical Co., Ltd. (Jinan, China). Fluorescein sodium was obtained from Alcon Laboratories, Inc. (Fort Worth, Texas, USA). The TUNEL kit was purchased from Roche (Basel, Switzerland).

\section{Animal}

Forty 22 -week-old male $\mathrm{db} / \mathrm{db}$ mice and 10 wild-type mice were obtained from The Model Animal Research Center of Nanjing University and raised in a temperaturecontrolled facility (temperature: $22 \pm 1{ }^{\circ} \mathrm{C}$; humidity: $60 \%$ ) with a $14 \mathrm{~h} \mathrm{light} / 10 \mathrm{~h}$ dark cycle in mouse cages. Ten wildtype mice and $\mathrm{db} / \mathrm{db}$ mice were divided into five groups randomly ( $\mathrm{n}=10 /$ group): control group, model group, GRg1 (25 and $50 \mathrm{mg} / \mathrm{kg}$ ) groups and dobesilate group. Weights were measured once a week. The GRg1 and dobesilate groups were orally administrated 25,50 and $225 \mathrm{mg} / \mathrm{kg} /$ day, respectively, for two months. The other two groups were administered the same volume of saline. The dose was chosen based on a published paper (15) and our pilot study. The retinas and blood samples were dissected from the eye after 8 weeks of GRg1 treatment.

\section{Blood glucose, blood lipids and glycosylated bemoglobin (HbA1c) detection}

At the end of GRg1 treatment, the mice were fasted $12 \mathrm{~h}$ and blood glucose was measured at the tail by a glucose meter (Roche, Switzerland). Mouse serum was acquired from the inner canthus. Serum lipids and HbAlc were determined by using commercial kits and a Beckman AU480 biochemical auto-analyser (Fullerton, CA, USA).

\section{Fundus fluorescein angiography (FFA)}

Changes in mouse retinal vascular permeability were tested 
by FFA. The mice were anaesthetized and the eyes were dilated with $1 \%$ tropicamide eye drops. Then, the eyeball was covered by carbomer. $2.5 \%$ fluorescein sodium $(0.1 \mathrm{~mL}$, i.p.) was given. After $2 \mathrm{~min}$, fundus examinations were performed and captured using a digital fundus camera (Retinal Imaging System, OptoProbe Research Ltd., Burnaby, Canada). The fluorescence intensity of the captured photographs from every group was analysed using AngioTool software (version 1.8.0) for statistical analysis.

\section{Optical coberence tomography (OCT)}

The retinal thicknesses of the mice were measured using a retinal imaging system (isOCT, 4D-ISOCT Optoprobe, Burnaby, Canada). The mice were anaesthetized, and $1 \%$ tropicamide eye drops were used to dilate eyes. Then, the eyeball was covered by carbomer. Images were acquired, and retinal thicknesses were calculated with software (version 2.0) from OptoProbe Research Ltd.

\section{HE staining}

The mice were euthanized by cervical dislocation, and the retina was quickly isolated and fixed in eye fixation fluid, embedded in paraffin and sectioned $(5 \mu \mathrm{m})$. The eye paraffin sections were dyed with haematoxylin and eosin. Images were captured by a digital slide image scanning and analysis system (Aperio CS2, Leica, Germany).

\section{TUNEL assay}

The TUNEL assay was performed according to our previous study (17). Briefly, paraffin sections were deparaffined and repaired by proteinase $\mathrm{K}$. Then, the sections were incubated with TdT enzyme and d UTP for $2 \mathrm{~h}$ at $37^{\circ} \mathrm{C}$, washed by PBS. The nuclei were stained with $\mathrm{DAB}$. Images were captured and analysed by a digital slide image scanning and analysis system (Aperio CS2, Leica, Germany).

\section{Immunofluorescence assay}

Retinal cryosections were prepared based on our previous method (17). The retinal sections were fixed with $4 \%$ paraformaldehyde, incubated with $1 \%$ Triton X-100 (Sigma, USA), and then incubated with anti-Bax, Bcl-2 and Caspase- 9 antibodies at $4{ }^{\circ} \mathrm{C}$ for $12 \mathrm{~h}$. The specimens were subsequently washed with PBS. Then, the appropriate secondary antibodies were added and incubated at $37{ }^{\circ} \mathrm{C}$ for $1 \mathrm{~h}$. The specimens were then stained with DAPI to label the nuclei, followed by the placement of a coverslip. Thereafter, the specimens were captured using fluorescence microscopy (Carl Zeiss, Göttingen, Germany).

\section{Western blotting}

At the end of GRg1 treatment, mouse retinas were acquired and added to RIPA lysis buffer including protease inhibitor. $10 \%$ or $12 \%$ sodium dodecyl sulfate polyacrylamide gel electrophoresis (SDS-PAGE) were applied to separate the cell extracts, then the proteins were transferred onto a nitrocellulose membrane. The membrane was incubated $12 \mathrm{~h}$ with $1: 1,000$-diluted primary antibodies at $4{ }^{\circ} \mathrm{C}$, followed by HRP-conjugated secondary antibodies at room temperature. The blots were captured, and analyses were performed using Gel Pro software (Media Cybernetics, Rockville, MD, USA) according to our previous study (18).

\section{Statistical analysis}

GraphPad Prism 6.0 software (San Diego, CA, USA) was applied for all analyses. Data are expressed as the mean \pm SD. Multigroup comparisons were analysed by one-way analysis of variance (ANOVA) followed by Tukey's post hoc test method. Student's unpaired $t$-test were performed to compare differences between two groups. A $\mathrm{P}<0.05$ was regarded as a statistical significance.

\section{Results}

\section{GRg1 reduced blood glucose and HbA1c levels}

Epidemiological studies demonstrated the promoting effects of hyperglycemia, dyslipidemia and a high bodymass index on the incidence and progression of DR (3). Thus, we first detected blood glucose levels. As given in Figure 1A, GRg1 $(50 \mathrm{mg} / \mathrm{kg})$ treatment significantly reduced fasting blood glucose levels compared with those of the model group. We also detected HbA1c, another gold standard detection method of blood glucose, and the results indicated that GRg1 also obviously decreased HbA1c levels (Figure 1B). GRg1 exhibited no significant effects on body weight, as indicated in Figure 1C. Taken together, GRg1 reduced diabetic mouse blood glucose levels after 2 months of treatment. 

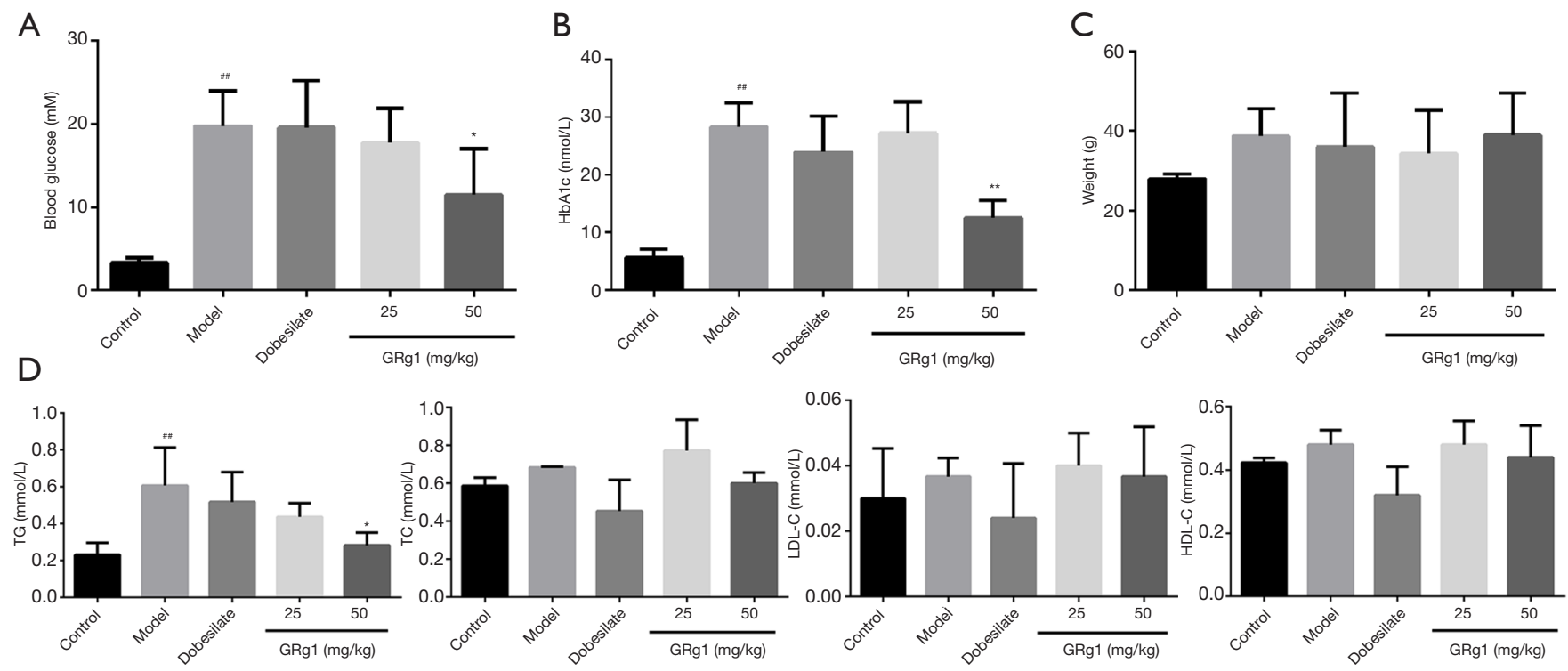

Figure 1 Weight, blood glucose, HbA1c and blood lipid levels of mice after 8 weeks of GRg1 administration. (A) Fasting blood glucose in all mice; (B) HbA1c expression level in all mice; (C) body weight of all mice; (D) TG, TC, LDL-C and HDL-C levels in all mice. The data are shown as the mean $\pm \mathrm{SD}$ after normalization to the control ( $\mathrm{n}=6$ /group). ${ }^{\# \#}, \mathrm{P}<0.01$ vs. the control group; ${ }^{*}, \mathrm{P}<0.05,{ }^{* *}, \mathrm{P}<0.01$ vs. the model group.

A

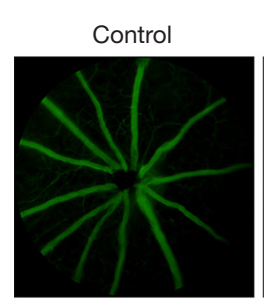

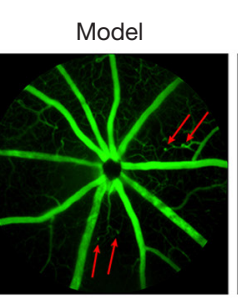
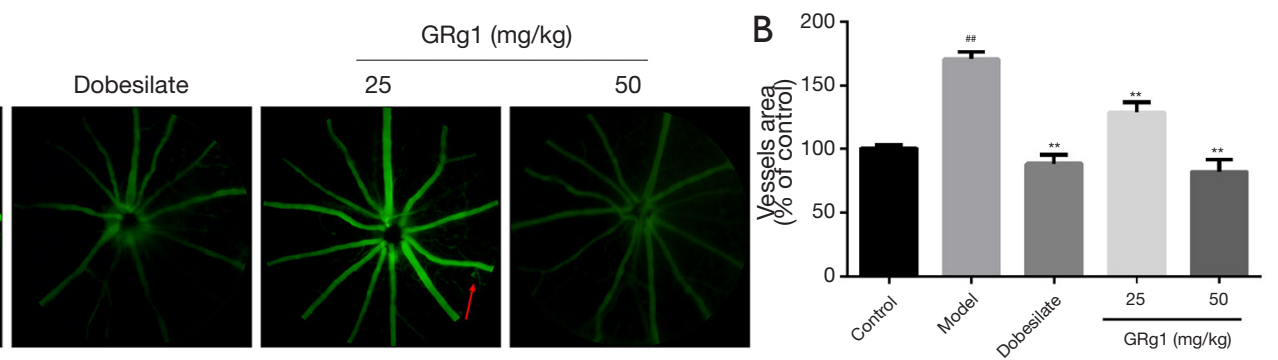

Figure 2 GRg1 ameliorated diabetes-induced retinal permeability. (A) Representative images of retinal FFA. Permeability in the retina (red arrows); (B) statistical results of FFA. The data are shown as the mean $\pm \mathrm{SD}$ after normalization to the control ( $\mathrm{n}=6 /$ group). ${ }^{\#}, \mathrm{P}<0.01$ s. the control group; ** $\mathrm{P}<0.05$ vs. the model group.

\section{GRg1 decreased blood lipid levels}

We tested the blood lipid levels of all mice. As shown in Figure 1D, GRg1 markedly reduced TG levels compared with those of the model group, although it had no obvious effects on TC, LDL-C or HDL-C levels.

\section{GRg1 ameliorated diabetes-induced retinal permeability}

Retinal permeability is one of the pathological events in early DR (19). To investigate changes in retinal vessels, we performed fluorescein angiography at two months of GRg1 administration in all groups. As shown in Figure 2, GRg1 markedly reduced diabetic mouse retinal permeability, which suggests that GRg1 could ameliorate DR.

\section{GRg1 protected against retinal GCL and inner nuclear layer (INL) injury}

To understand whether GRg1 has protective effects on diabetic mouse retinal damage, we detected retinal layer thickness and histological features. As exhibited in Figure 3A, the retinas from the control group showed normal 
A

Control

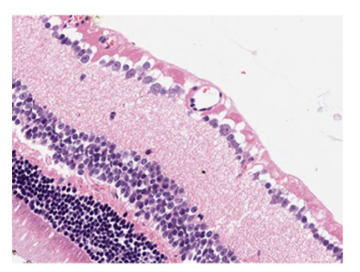

B

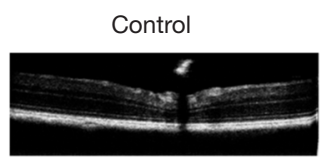

C

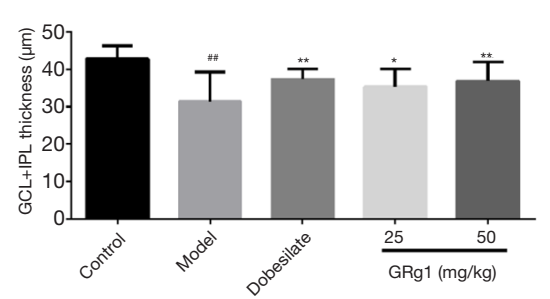

Model

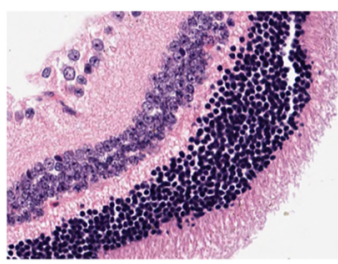

Model

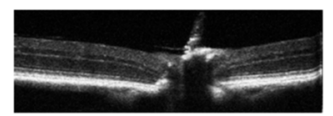

Dobesilate

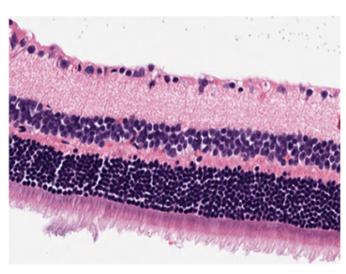

Dobesilate

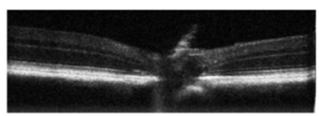

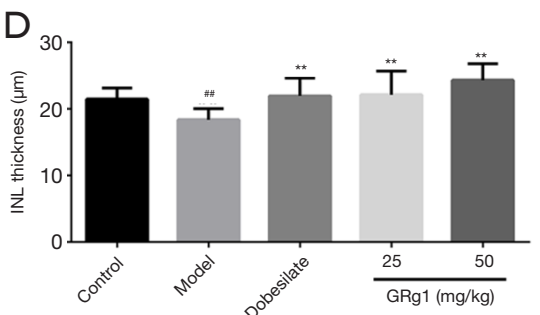

GRg1 $(\mathrm{mg} / \mathrm{kg})$

$25 \quad 50$
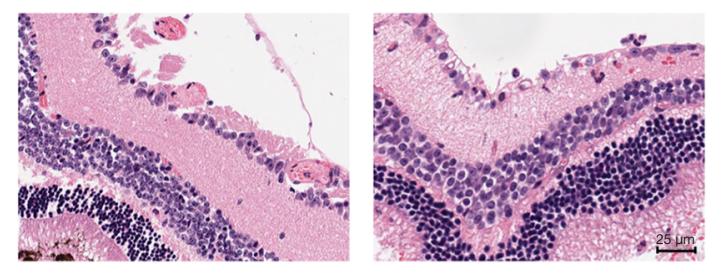

GRg1 (mg/kg)
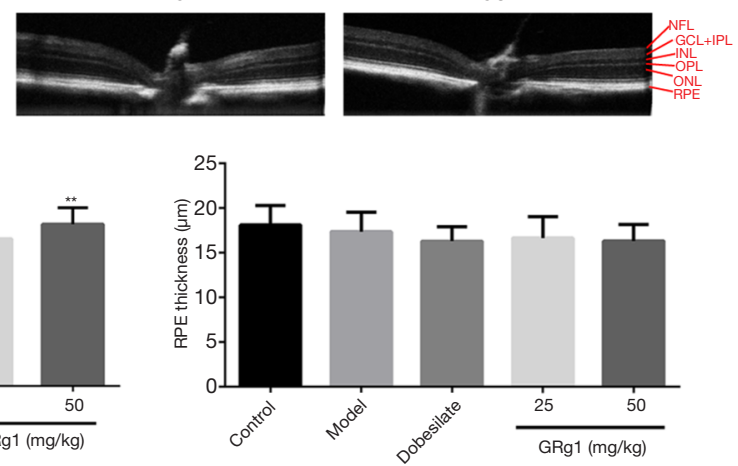

Figure 3 GRg1 protected against retinal INL injury. (A) Representative HE-stained images of sections from the retinas of each group $(\times 200)$; (B) representative images of retinal OCT; (C) statistic results of GCL+IPL and RPE in each group; (D) statistical results of the INL in each group. The data are shown as the mean $\pm \mathrm{SD}$ after normalization to the control ( $\mathrm{n}=6 /$ group). ${ }^{\text {\#\# }}, \mathrm{P}<0.01$ vs. the control group; * $\mathrm{P}<0.05$, **, $\mathrm{P}<0.01$ vs. the model group. NFL, nerve fibre layer; GCL, ganglion cell layer; IPL, inner plexiform layer; INL, inner nuclear layer; OPL, outer plexiform layer; ONL, outer nuclear layer; RPE, retinal pigment epithelium.

structures. However, the model group showed morphologic changes in the retinas, such as marked disorganization of the retinal layers, degeneration and reduced numbers of retinal cells in the GCL and INL. A mild improvement in the morphology was evident in the retinas of the GRg1 treatment groups. As shown in Figure 3B,C,D, the GCL and INL thickness in the model group mice was thinner than that in the control group, whereas GRg1 treatment significantly improved it the thickness. Moreover, GRg1 had no obvious effects on the thickness of RPE (Figure 3D), which are also a vulnerable layer in DR (20). Taken together, these results indicate that GRg1 prevents retinal injury in diabetic mice.

\section{GRg1 decreased retinal GCL and INL cell apoptosis}

Cell apoptosis is a strong marker in the pathogenesis of DR (21). To detect the effects of GRg1 on retinal injury, we evaluated retinal cell apoptosis using an immunofluorescence assay (Figure 4). As indicated in Figure 4A,C, mice in the model group exhibited significantly higher expression levels of the pro-apoptotic proteins Bax and Caspase-9 compared with those of the control group, whereas expression of the anti-apoptotic protein Bcl-2 was reduced. However, GRg1 markedly reduced Bax, and Caspase-9 expression levels and increased Bcl-2 expression levels in the INL. To further verify the above findings, we measured TUNEL-positive cells. As shown in Figure 4B,D, the retinas in the model group exhibited increased numbers of TUNEL-positive cells in the INL. In comparison, the retinas in the GRg1 group contained fewer TUNEL-positive cells, which was consistent with a previous study (22).

\section{GRg1 modulated apoptosis-related protein expression level in retina}

Next, we further measured apoptosis-related protein expression levels in the retina. As indicated in Figure 5, 
A
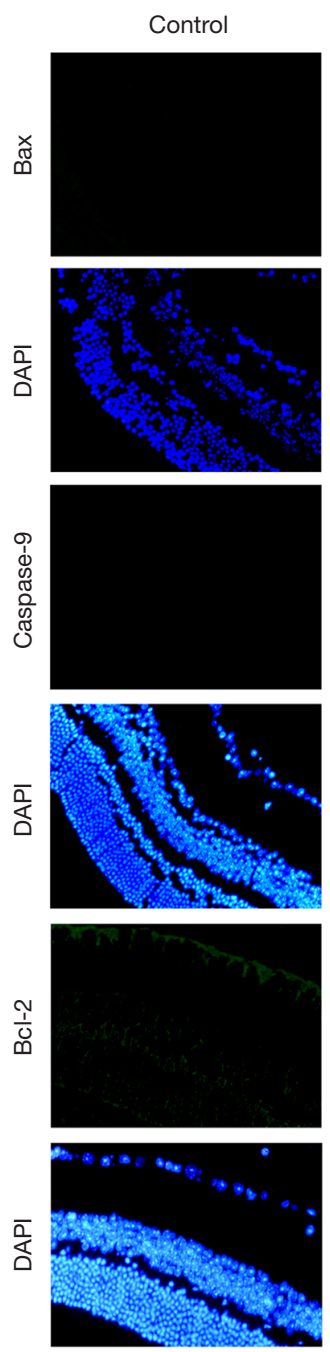

B
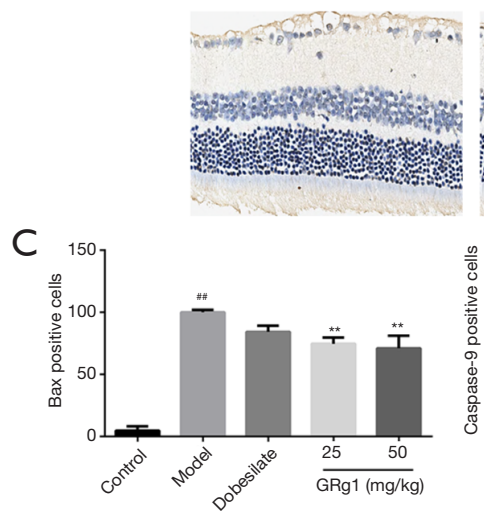
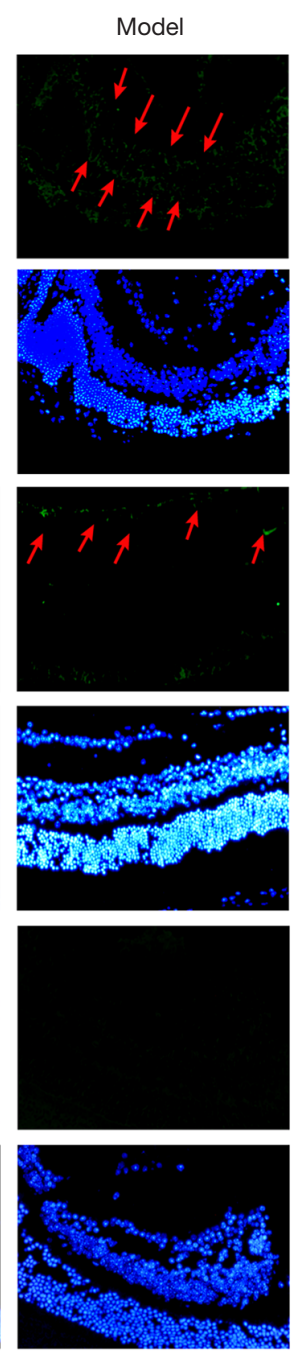

Model

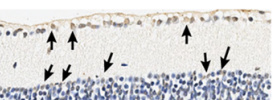

$1+t-1 \downarrow$

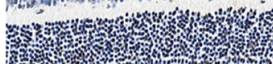

Dobesilate
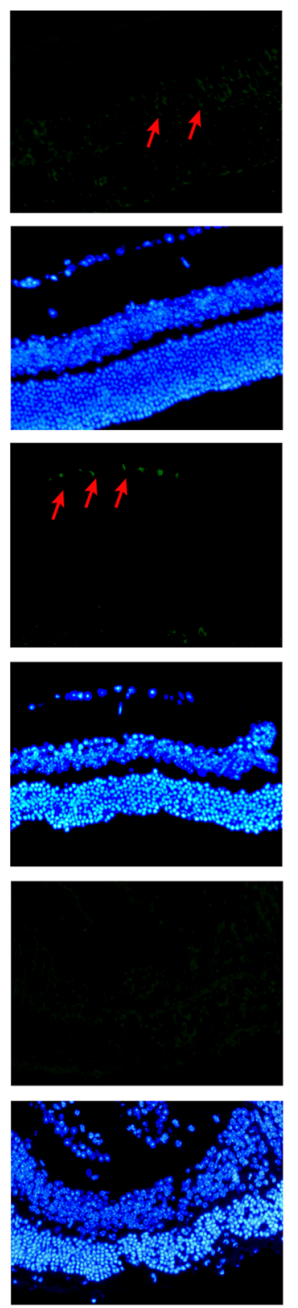

Dobesilate
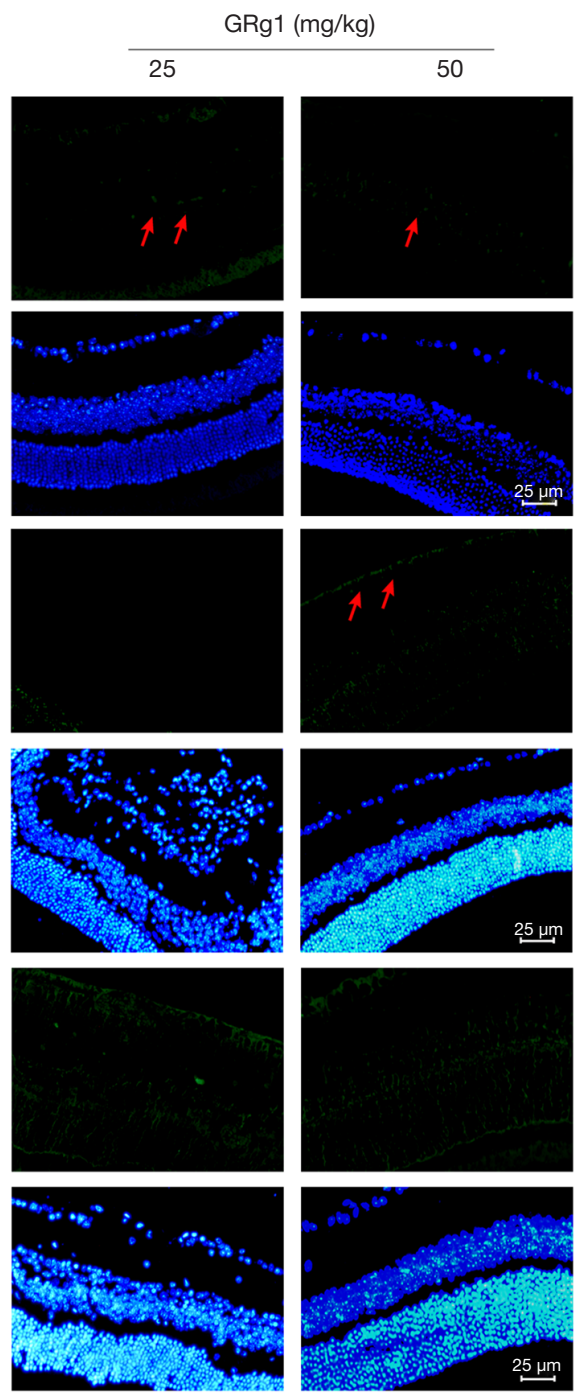

GRg1 (mg/kg)
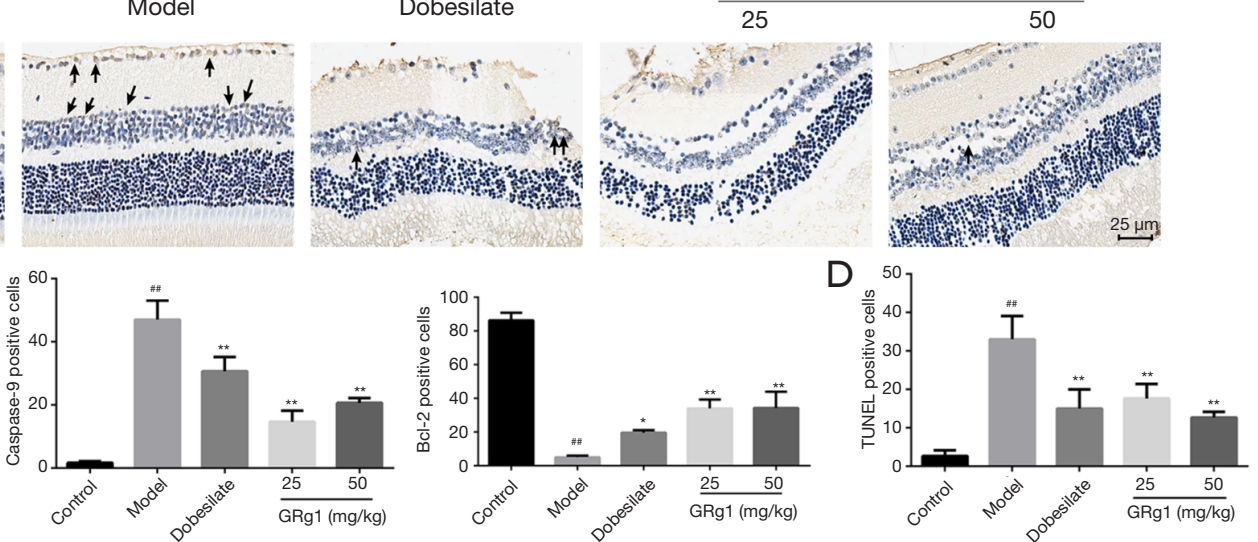

Figure 4 GRg1 decreased retinal INL cell apoptosis. (A) Representative images of sections from the retinas of each group stained with Bax, Caspase-9 and Bcl-2 (×200); (B) representative images of retinas from TUNEL assays $(\times 200)$. Apoptotic cells in the retina (black arrows); (C) statistical results of Bax-, Caspase-9- and Bcl-2-positive cells; (D) statistical results of TUNEL-positive cells. The data are shown as the mean $\pm \mathrm{SD}$ after normalization to the control (n=6/group). ${ }^{\#}, \mathrm{P}<0.01$ vs. the control group; $, \mathrm{P}<0.05,{ }^{* *}, \mathrm{P}<0.01$ vs. the model group. 
A

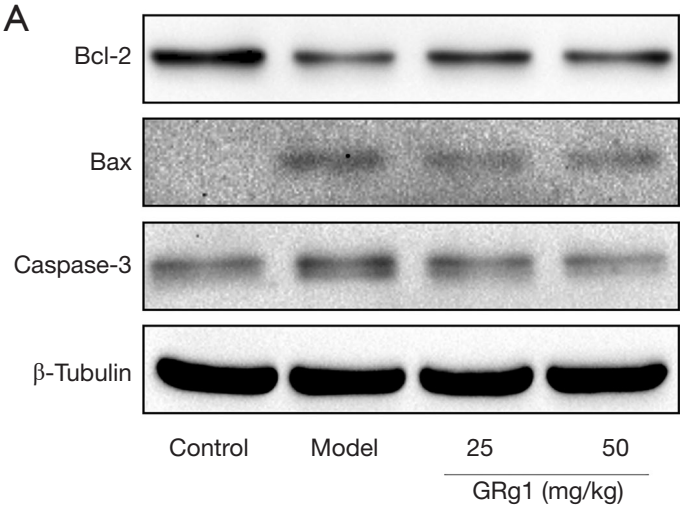

B

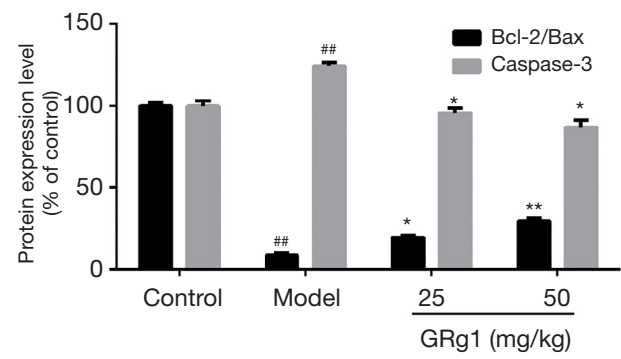

Figure 5 GRg1 modulated apoptosis-related protein expression levels in the retina. (A) Representative images of Bax, Caspase-9, Bcl-2 and $\beta$-Tubulin by western blotting; (B) statistical result of Bcl-2/Bax and Caspase- 9 expression levels. The data are shown as the mean \pm SD after normalization to the control ( $\mathrm{n}=6$ /group). ${ }^{\# \#}, \mathrm{P}<0.01$ vs. the control group; ${ }^{*}, \mathrm{P}<0.05$, ${ }^{* *}, \mathrm{P}<0.01$ vs. the model group.

GRg1 obviously increased the Bcl-2/Bax ratio and decreased the caspase-3 expression level. Taken together, GRg1 reduced retinal injury by ameliorating cell apoptosis in diabetic mice.

\section{Discussion}

With the increasing morbidity of diabetes, the number of DR patients is also increasing rapidly. Although there are several effective drugs for DR treatment, they are still not enough because they mainly focus late DR and have inevitable side effects. Thus, we must find new safe and effective drugs for use in the early disease to address unmet medical needs. Based on a previous study, GR1, one of the main components in ginseng, possesses a variety of pharmacological properties, including anti-diabetic (15), neuro-protective (23) and anti-apoptotic effects (24). In this study, we found that GRg1 protects the diabetic retina at the early stage of diabetes.

High blood glucose and blood lipids are two direct factors during diabetes development (19). Our data indicated that GRg1 exhibited strong blood glucose and TG reducing effects. Fundus fluorescence angiography is a reliable method to detect retinal vascular leakage (25), which is an early pathological change in DR. Our results indicated that GRg1 obviously reduced vascular permeability in a dose-dependent manner, which was consistent with a previous study (26).

In addition to glucose reduction and permeability inhibition, we also demonstrated that GRg1 reduced pathological changes in the retinal layers. HE results showed that GRg1 significantly ameliorated retinal edema and disordered cell arrangement. Moreover, we also demonstrated that GRg1 treatment significantly improved the thickness of the GCL and INL, where there are many retinal cells, such as ganglion cells, pericytes, endothelial cells, and müller cells (3). Our results strongly showed that a thinner GCL and INL could be a characteristic for early DR screening, which was consistent with a previous study (7).

Neuroprotective and anti-apoptotic agents have been regarded as excellent DR treatments (27). GRg1 prevents hyperphosphorylated tau-induced synaptic neurodegeneration of RGCs via activation of IRS-1/Akt/ GSK3 $\beta$ signalling in the early phase of DR (23). GRg1 ameliorates ER stress-induced apoptosis in diabetic rats (16). Thus, we further investigated whether the retinal protective effects of GRg1 are related to its anti-apoptotic actions. As expected, our data showed that GRg1 decreased the expression of the pro-apoptotic proteins Caspase-9 and Bax and increased the expression of the anti-apoptotic protein Bcl-2 in the GCL and INL, which was also demonstrated by western blotting. Taken together, these results suggest that GRg1 protects against retinal injury by inhibiting retinal cell apoptosis, especially in GCL and INL cells.

\section{Conclusions}

Our data strongly suggest the anti-apoptotic effects of GRg1 in the diabetic retina for the first time. We also show data indicating that the actions of GRg1 occur through the inhibition of retinal cell apoptosis in the GCL and INL. However, there still have some limitations to our study. 
We focused on the retinal protective effects of GRg1 and ignored the pro-apoptotic factors, such as anti-oxidative and anti-inflammatory factors. Future studies are needed to further elucidate the exact mechanisms by which GRg1 protects the diabetic retina.

\section{Acknowledgments}

Funding: This study was financially supported by the Major Scientific and Technological Special Project "Drug Innovation Major Project" (No. 2018ZX09711001-009) and the National Natural Science Foundation of China (No. 81703745 and No. 81891012).

\section{Footnote}

Conflicts of Interest: YL serves an unpaid section editor of Annals of Translational Medicine from Oct 2019 to Sep 2020. The other authors have no conflicts of interest to declare.

Etbical Statement: The authors are accountable for all aspects of the work in ensuring that questions related to the accuracy or integrity of any part of the work are appropriately investigated and resolved. All animal care procedures and interventions were consistent with the Guidelines and Policies for Animal Surgery provided by the Chinese Academy of Medical Sciences and Peking Union Medical College, Beijing, China [SYXK(Jing) 2017-0020] and admitted by the Institutional Animal Use and Care Committee.

Open Access Statement: This is an Open Access article distributed in accordance with the Creative Commons Attribution-NonCommercial-NoDerivs 4.0 International License (CC BY-NC-ND 4.0), which permits the noncommercial replication and distribution of the article with the strict proviso that no changes or edits are made and the original work is properly cited (including links to both the formal publication through the relevant DOI and the license). See: https://creativecommons.org/licenses/by-nc-nd/4.0/.

\section{References}

1. Federation ID. IDF Diabetes Atlas. 8th ed. 2017. Available online: http://wwwdiabetesatlasorg

2. Pearce I, Simó R, Lövestam-Adrian M, et al. Association between diabetic eye disease and other complications of diabetes: Implications for care. A systematic review. Diabetes Obes Metab 2019;21:467-78.

3. Antonetti DA, Klein R, Gardner TW. Diabetic retinopathy. N Engl J Med 2012;366:1227-39.

4. Belokopytov M, Shulman S, Dubinsky G, et al. Ameliorative effect of NAP on laser-induced retinal damage. Acta Ophthalmol 2011;89:e126-31.

5. Sun JK, Wang PW, Taylor S, et al. Durability of Diabetic Retinopathy Improvement with As-Needed Ranibizumab: Open-Label Extension of RIDE and RISE Studies. Ophthalmology 2019;126:712-20.

6. Gharbiya M, Bruscolini A, Sacchetti M, et al. In vivo antivascular endothelial growth factor treatment induces corneal endothelium apoptosis in rabbits through changes in p75NTR-proNGF pathway. J Cell Physiol 2018;233:8874-83.

7. Sohn EH, van Dijk HW, Jiao C, et al. Retinal neurodegeneration may precede microvascular changes characteristic of diabetic retinopathy in diabetes mellitus. Proc Natl Acad Sci U S A 2016;113:E2655-64.

8. Thounaojam MC, Powell FL, Patel S, et al. Protective effects of agonists of growth hormone-releasing hormone (GHRH) in early experimental diabetic retinopathy. Proc Natl Acad Sci U S A 2017;114:13248-53.

9. Vasant More S, Kim IS, Choi DK. Recent Update on the Role of Chinese Material Medica and Formulations in Diabetic Retinopathy. Molecules 2017. doi: 10.3390/ molecules22010076.

10. Zhang HW, Zhang H, Grant SJ, et al. Single herbal medicine for diabetic retinopathy. Cochrane Database Syst Rev 2018;12:CD007939.

11. Zhang T, Mei X, Ouyang H, et al. Natural flavonoid galangin alleviates microglia-trigged blood-retinal barrier dysfunction during the development of diabetic retinopathy. J Nutr Biochem 2019;65:1-14.

12. Mei X, Zhang T, Ouyang H, et al. Scutellarin alleviates blood-retina-barrier oxidative stress injury initiated by activated microglia cells during the development of diabetic retinopathy. Biochem Pharmacol 2019;159:82-95.

13. Fan C, Qiao Y, Tang M. Notoginsenoside R1 attenuates high glucose-induced endothelial damage in rat retinal capillary endothelial cells by modulating the intracellular redox state. Drug Des Devel Ther 2017;11:3343-54.

14. Bi S, Ma X, Wang Y, et al. Protective Effect of Ginsenoside Rg1 on Oxidative Damage Induced by Hydrogen Peroxide in Chicken Splenic Lymphocytes. Oxid Med Cell Longev 2019;2019:8465030.

15. Liu Q, Zhang FG, Zhang WS, et al. Ginsenoside Rg1 
Inhibits Glucagon-Induced Hepatic Gluconeogenesis through Akt-FoxO1 Interaction. Theranostics 2017;7:4001-12

16. Yu H, Zhen J, Yang Y, et al. Ginsenoside Rg1 ameliorates diabetic cardiomyopathy by inhibiting endoplasmic reticulum stress-induced apoptosis in a streptozotocin-induced diabetes rat model. J Cell Mol Med 2016;20:623-31.

17. Luo Y, Dong X, Yu Y, et al. Total aralosides of aralia elata (Miq) seem (TASAES) ameliorate nonalcoholic steatohepatitis by modulating IRE1alpha-mediated JNK and NF-kappaB pathways in ApoE-/- mice. J Ethnopharmacol 2015;163:241-50.

18. Luo $\mathrm{Y}$, Meng X, Zhou $\mathrm{P}$, et al. Elatoside $\mathrm{C}$ protects against ox-LDL-induced HUVECs injury by FoxO1-mediated autophagy induction. Biochim Biophys Acta Mol Basis Dis 2017;1863:1654-65.

19. Roy S, Kern TS, Song B, et al. Mechanistic Insights into Pathological Changes in the Diabetic Retina: Implications for Targeting Diabetic Retinopathy. Am J Pathol 2017;187:9-19.

20. Du W, An Y, He X, et al. Protection of Kaempferol on Oxidative Stress-Induced Retinal Pigment Epithelial Cell Damage. Oxid Med Cell Longev 2018;2018:1610751.

21. Volpe CMO, Villar-Delfino PH, Dos Anjos PMF, et al.

Cite this article as: Gao Y, Ji Y, Luo Y, Sun J, Sun G, Sun X. Ginsenoside $\operatorname{Rg} 1$ prevents early diabetic retinopathy via reducing retinal ganglion cell layer and inner nuclear layer cell apoptosis in db/db mice. Ann Transl Med 2020;8(5):232. doi: 10.21037/atm.2019.12.155
Cellular death, reactive oxygen species (ROS) and diabetic complications. Cell Death Dis 2018;9:119.

22. Liu J, Bhuvanagiri S, Qu X. The protective effects of lycopus lucidus turcz in diabetic retinopathy and its possible mechanisms. Artif Cells Nanomed Biotechnol 2019;47:2900-8.

23. Ying Y, Zhang YL, Ma CJ, et al. Neuroprotective Effects of Ginsenoside Rg1 against Hyperphosphorylated Tau-Induced Diabetic Retinal Neurodegeneration via Activation of IRS-1/Akt/GSK3 $\beta$ Signaling. J Agric Food Chem 2019;67:8348-60.

24. Shi Q, Chen X, Sun G, et al. Ginsenoside Rg1 protects human retinal pigment epithelial ARPE-19 cells from toxicity of high glucose by up-regulation of miR-26a. Life Sci 2019;221:152-8.

25. Ip MS, Zhang J, Ehrlich JS. The Clinical Importance of Changes in Diabetic Retinopathy Severity Score. Ophthalmology 2017;124:596-603.

26. Liu L, Jiang Y, Steinle JJ. Glycyrrhizin Protects the Diabetic Retina against Permeability, Neuronal, and Vascular Damage through Anti-Inflammatory Mechanisms. J Clin Med 2019. doi: 10.3390/jcm8070957.

27. Mrugacz M, Bryl A, Bossowski A. Neuroretinal Apoptosis as a Vascular Dysfunction in Diabetic Patients. Curr Neuropharmacol 2016;14:826-30. 